\title{
Dufour and Soret Effect on Steady MHD Flow in Presence of Heat Generation and Magnetic Field past an Inclined Stretching Sheet
}

\author{
Md Enamul Karim¹, Md Abdus Samad², Md Maruf Hasan ${ }^{1}$ \\ ${ }^{1}$ Department of Mathematics, Comilla University, Comilla, Bangladesh \\ ${ }^{2}$ Department of Mathematics, University of Dhaka, Dhaka, Bangladesh \\ Email: ekarim_du@yahoo.com
}

Received April 25, 2012; revised May 30, 2012; accepted June 18, 2012

\begin{abstract}
An analysis of two-dimensional steady magneto-hydrodynamic free convection flow of an electrically conducting, viscous and incompressible fluid past an inclined stretching porous plate in the presence of a uniform magnetic field and thermal radiation with heat generation is made. Both the Dufour and Soret effects are considered for a hydrogen-air mixture as the non-chemically reacting fluid pair. The equations governing the flow, temperature and concentration fields are reduced to a system of joined non-linear ordinary differential equations by similarity transformation. Non-linear differential equations are integrated numerically by using Nachtsheim-Swigert shooting iteration technique along with sixth order Runge-Kutta integration scheme. Finally the significance of physical parameters which are of engineering interest are examined both in graphical and tabular form.
\end{abstract}

Keywords: Dufour Effect; Magnetic Field; MHD; Nusselt Number and Soret Number

\section{Introduction}

Laminar boundary layer flow, a significant type of flow, in presence of magnetic field and radiation over a moving continuous and linearly stretched surface has been receiving wide attention due to its applications in engineering, electro-chemistry, Gorla [1], Chin [2] and polymer processing, Erickson [3]. For examples, materials manufactured by aerodynamic extrusion processes and heat-treated materials traveling between a feed roll and a wind-up roll or on a conveyor belt possess the characteristics of a moving continuous stretching surface. Moreover lots of metallurgical processes occupy the system of cooling of continuous strips or filaments by drawing them through a quiescent fluid and that in the process of drawing, these strips are sometimes stretched. Another important matter is that the final product depends to a great extent on the rate of cooling. By drawing such strips in an electrically conducting fluid subjected to a magnetic field, the rate of cooling can be controlled and a final product of desired characteristics can be achieved. The study of heat and mass transfer is necessary for determining the quality of the final product, Samad and Mohebujjaman [4].

Sakiadis [5] analyzed the boundary layer flow over a solid surface moving with a constant velocity. Cess [6] examined the effect of thermal radiation on absorbing emitting gray fluids with black vertical plate. The above investigations having a definite bearing on the problem of a polymer sheet extruded continuously from a dye. It is usually assumed that the sheet is inextensible, but situations may arise in the polymer industry in which it is necessary to deal with a stretching plastic sheet, as noted by Crane [7]. Sparrow [8] explained a parameter named Rosseland approximation to describe the radiation heat flux in the energy equation in his book. Soundalgekar et al. [9] made analytical study on the MHD forced and free convection flow past a vertical porous plate. The flow field of a stretching wall with a power-law velocity variation was discussed by Banks [10]. McLeod and Rajagopal [11] investigated the uniqueness of the flow of a Navier Stokes fluid due to a linear stretching boundary. Chen and Char [12] studied the suction and injection on a linearly moving plate subject to uniform wall temperature and heat flux. By using the Rosseland diffusion approximation [8], a study of the unsteady mixed convection flow of an optically dense viscous incompressible fluid past a heated vertical plate with a free uniform stream velocity and surface temperature was made by Hossain and Takhar [13].

The thermal-diffusion (Soret) effect, for instance, has been utilized for isotope separation, and in mixtures be- 
tween gases with very light molecular weight $(\mathrm{Hz}, \mathrm{He})$ and of medium molecular weight $(\mathrm{Nz}$, air) the diffusion-thermo (Dufour) effect was found to be of a considerable magnitude such that it cannot be ignored, described by Eckert and Drake [14] in their book. Alabraba et al. [15] studied the interaction of mixed convection with thermal radiation in laminar boundary layer flow taking into account the binary chemical reaction and Soret-Dufour effects. Postelnicu [16] studied numerically the influence of a magnetic field on heat and mass transfer by natural convection from vertical surfaces in porous media considering Soret and Dufour effects. Alam and Rahman [17] discovered the Dufour and Soret effect on unsteady MHD flow in a porous medium. Very recently Karim et al. [18] discussed the thermal effect on MHD flow.

The present paper is the investigation of the Dufour and Soret effects of an electrically conducting viscous incompressible fluid interaction with thermal radiation on the flow over an inclined linearly stretched sheet in the presence of heat and mass transfer permitted by a transversely applied uniform magnetic field with heat generation taking into account the Rosseland diffusion approximation. The investigation is based on known similarity analysis and the local similarity solutions are obtained numerically.

\section{Mathematical Analysis}

A steady-state two-dimensional heat and mass transfer flow of an electrically conducting viscous incompressible fluid along an isothermal stretching permeable inclined sheet with an angle $\alpha$ to the vertical embedded in a porous medium with heat generation/absorption is considered. A strong magnetic field is applied in the $y$-axis direction. Here the effect of the induced magnetic field is neglected in comparison to the applied magnetic field. The electrical current flowing in the fluid gives rise to an induced magnetic field if the fluid were an electrical insulator, but here we have taken the fluid to be electrically conducting. Hence, only the applied magnetic field of strength $B_{0}$ plays a role which gives rise to magnetic forces $F=\frac{\sigma B_{0}^{2} u}{\rho}$ in $x$-direction, $\sigma$ where is the electrical conductivity assumed to be directly proportional to the $x$-translational velocity $(u)$ of the fluid found by Helmy [19] and $\rho$ is the density of the fluid. We also bring into account the effect of temperature dependent volumetric heat generation $\left(\mathrm{W} / \mathrm{m}^{3}\right)$, in the flow region that is given by Vajravelu and Hadjinicolaou [20] as

$$
q^{\prime \prime \prime}=Q_{0}\left(T-T_{\infty}\right), T \geq T_{\infty}
$$

where $Q_{0}$ is the heat generation. Two equal and opposite forces are introduced along the $x$-axis so that the sheet is stretched keeping the origin fixed as shown in Figure 1.

The fluid is considered to be gray, absorbing emitting radiation but non-scattering medium and the Rosseland approximation is used to describe the radiation heat flux in the energy equation. The radiative heat flux in the $x$-direction is negligible to the flux in the $y$-direction. The plate temperature and concentration are initially raised to $T_{w}$ and $C_{w}$ respectively which are thereafter maintained constant. The ambient temperature of the flow is $T_{\infty}$ and the concentration of the uniform flow is $C_{\infty}$.

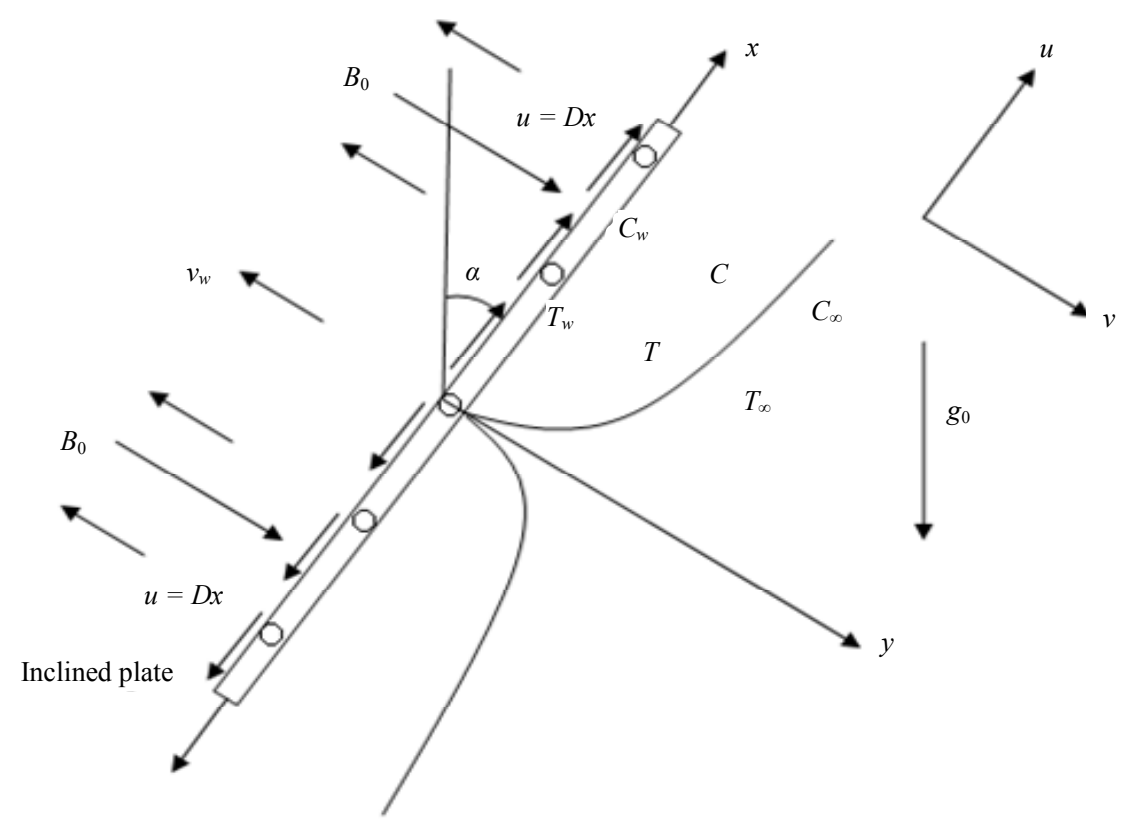

Figure 1. Flow configuration and coordinate system. 
Under the usual boundary layer and Boussinesq approximations and using the Darcy-Forchhemier model, the flow and heat transfer in the presence of radiation are governed by the following equations.

Continuity Equation

$$
\frac{\partial u}{\partial x}+\frac{\partial v}{\partial y}=0
$$

Momentum Equation

$$
\begin{aligned}
& u \frac{\partial u}{\partial x}+v \frac{\partial u}{\partial y}=v \frac{\partial^{2} u}{\partial y^{2}}+g_{0} \beta\left(T-T_{\infty}\right) \times \cos \alpha \\
& +g_{0} \beta^{*}\left(C-C_{\infty}\right) \cos \alpha-\frac{\sigma B_{0}^{2} u}{\rho}-\frac{v u}{k}-\frac{b u^{2}}{k}
\end{aligned}
$$

Energy Equation

$$
\begin{aligned}
& u \frac{\partial T}{\partial x}+v \frac{\partial T}{\partial y}=\frac{\kappa}{\rho c_{p}} \frac{\partial^{2} T}{\partial y^{2}}+\frac{D_{m} K_{T}}{c_{s} c_{p}} \frac{\partial^{2} C}{\partial C^{2}} \\
& +\frac{v}{c_{p}}\left(\frac{\partial u}{\partial y}\right)^{2}+\frac{Q_{0}}{\rho c_{p}}\left(T-T_{\infty}\right)-\frac{1}{\rho c_{p}} \frac{\partial q_{r}}{\partial y}
\end{aligned}
$$

Concentration Equation

$$
u \frac{\partial C}{\partial x}+v \frac{\partial C}{\partial y}=D_{m} \frac{\partial^{2} C}{\partial y^{2}}+\frac{D_{m} K_{T}}{T_{m}} \frac{\partial^{2} T}{\partial y^{2}}
$$

where $u$ and $v$ are the velocity components in the $x$ and $y$-directions respectively, $v$ is the kinematic viscosity, $g_{0}$ is the acceleration due to gravity, $\beta$ is the volumetric coefficient of thermal expansion, $T$ and $T_{\infty}$ are the fluid temperature within the boundary layer and in the free-stream respectively, while $C$ is the concentration of the fluid within the boundary layer, $\sigma$ is the electric conductivity, $B_{0}$ is the uniform magnetic field strength (magnetic induction), $\rho$ is the density of the fluid, $\kappa$ is the thermal conductivity of the fluid, $c_{p}$ is the specific heat at constant pressure, $Q_{0}$ is the volumetric rate of heat generation/absorption and $D_{m}$ is the chemical molecular diffusivity.

The corresponding boundary conditions for the model are

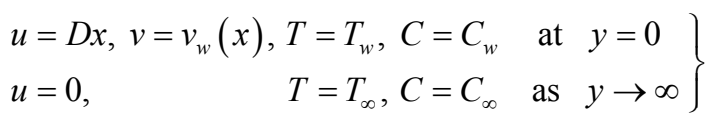

where $D(>0)$ is a constant, $v_{w}(x)$ is a velocity component at the wall having positive value to indicate suction, $T_{w}$ is the uniform sheet temperature and $C_{w}$ is the concentration of the fluid at the sheet.

By using Rosseland approximation $q_{r}$ takes the form

$$
q_{r}=-\frac{4 \sigma_{1}}{3 \kappa_{1}} \frac{\partial T^{4}}{\partial y}
$$

where $\sigma_{1}$ is the Stefan-Boltzmann constant and $\kappa_{1}$ is the mean absorption coefficient. It is assumed that the temperature differences within the flow are sufficiently small such that $T^{4}$ may be expressed as a linear function of temperature. This is accomplished by expanding $T^{4}$ in a Taylor series about $T_{\infty}$ and neglecting higher order terms, thus

$$
T^{4} \cong 4 T_{\infty}^{3} T-3 T_{\infty}^{4}
$$

Using Equations (6) \& (7), Equation (3) takes the form

$$
\begin{aligned}
u \frac{\partial T}{\partial x}+v \frac{\partial T}{\partial y} & =\frac{\kappa}{\rho c_{p}} \frac{\partial^{2} T}{\partial y^{2}}+\frac{D_{m} K_{T}}{c_{s} c_{p}} \frac{\partial^{2} C}{\partial C^{2}} \\
& +\frac{v}{c_{p}}\left(\frac{\partial u}{\partial y}\right)^{2}+\frac{Q_{0}}{\rho c_{p}}\left(T-T_{\infty}\right) \\
& +\frac{16 \sigma_{1} T_{\infty}^{3}}{3 \rho c_{p} \kappa_{1}} \frac{\partial^{2} T}{\partial y^{2}}
\end{aligned}
$$

\section{Similarity Analysis}

In order to obtain similarity solution for the problem under consideration, we may take the following suitable similarity variables

$$
\left.\begin{array}{l}
\eta=y \sqrt{\frac{D}{v}}, \psi=\sqrt{D v} \times f(\eta), \\
\theta(\eta)=\frac{T-T_{\infty}}{T_{w}-T_{\infty}}, \phi(\eta)=\frac{C-C_{\infty}}{C_{w}-C_{\infty}}
\end{array}\right\}
$$

where $\psi$ is the stream function, $\eta$ is the dimensionless distance normal to the sheet, $f$ is the dimensionless stream function, $\theta$ is the dimensionless fluid temperature and $\phi$ is the dimensionless concentration.

Since $u=\frac{\partial \psi}{\partial y}$ and $v=-\frac{\partial \psi}{\partial x}$ we have the velocity components from Equation (6) given by

$$
u=D x f^{\prime}(\eta) \text { and } v=-\sqrt{D v} f(\eta)
$$

where prime denotes the derivative with respect to $\eta$.

Now introducing the similarity variables from Equation (9) and using Equation (10), Equations (2) to (4) are reduced to the dimensionless equations given by

$$
\begin{gathered}
f^{\prime \prime \prime}+f f^{\prime \prime}+\gamma \cos \alpha \theta+\gamma^{*} \cos \alpha \phi \\
-\left(M+\frac{1}{D a \cdot \operatorname{Re}}\right) f^{\prime}-\left(1+\frac{F s}{D a}\right)\left(f^{\prime}\right)^{2}=0 \\
\theta^{\prime \prime}+P n f \theta^{\prime}+\operatorname{Pn} Q \theta+P n E c f^{\prime \prime 2}+P n D u \phi^{\prime \prime}=0 \\
\phi^{\prime \prime}+S c f \phi^{\prime}+\operatorname{SrSc} \theta^{\prime \prime}=0
\end{gathered}
$$

where $\gamma_{x}=\frac{G r_{x}}{\operatorname{Re}_{x}^{2}}=\frac{g_{0} \beta\left(T_{w}-T_{\infty}\right) x}{U^{2}}$ is the local buoyancy parameter, $\gamma_{x}^{*}=\frac{g_{0} \beta^{*}\left(C_{w}-C_{\infty}\right) x}{U^{2}}$ is the modified lo- 
cal buoyancy parameter, $M=\frac{\sigma B_{0}^{2}}{\rho D}$ is the magnetic field parameter, $D a=\frac{k}{x^{2}}$ is the local Darcy number, $\operatorname{Re}_{x}=\frac{x U}{v}$ is the Reynolds number, $F s=\frac{b}{x}$ is the Forchhemier number, $\operatorname{Pr}=\frac{\mu c_{p}}{\kappa}$ is the Prandtl number, $N=\frac{\kappa \kappa_{1}}{4 \sigma T_{\infty}^{3}}$ is the radiation parameter and $P n=\frac{3 N \mathrm{Pr}}{3 N+4}$, $Q=\frac{Q_{0}}{\rho c_{p} D}$ is the heat source $(Q>0) / \operatorname{Sink}(Q<0)$ parameter, $E c=\frac{U^{2}}{c_{p}\left(T_{w}-T_{\infty}\right)}$ is the Eckert number, $D u=\frac{D_{m} K_{T}\left(C_{w}-C_{\infty}\right)}{c_{s} c_{p} v\left(T_{w}-T_{\infty}\right)}$ is the Dufour number, $S_{c}=\frac{v}{D_{m}}$ is the Schmidt number and $S r=\frac{D_{m} K_{T}\left(T_{w}-T_{\infty}\right)}{T_{m} v\left(C_{w}-C_{\infty}\right)}$ is the Soret number.

The transformed boundary conditions are

$$
\left.\begin{array}{rl}
f=f_{w}, f^{\prime} & =1, \theta=1, \phi=1 \text { at } \eta=0 \\
f^{\prime} & =0, \theta=0, \phi=0 \text { as } \eta \rightarrow \infty
\end{array}\right\}
$$

where $f_{w}=-\frac{v_{w}}{\sqrt{D v}}$ is the suction parameter. The nonlinear ordinary differential Equations (8), (9) and (10) under the boundary conditions (11) are solved numerically for various values of the parameters entering into the problems.

Skin friction, rate of heat and mass transfer: The parameters of engineering interest for the present problem are the skin friction coefficient $\left(c_{f}\right)$, local Nusselt number $(\mathrm{Nu})$ and Sherwood number $(\mathrm{Sh})$ which indicate physically the wall shear stress, the rate of heat transfer and the local surface mass flux respectively. The dimensionless skin-friction coefficient, Nusselt number and Sherwood number for impulsively started plate are given by

$$
\begin{gathered}
C_{f}=\frac{2 \tau_{w}}{\rho U^{2}}=2\left(\operatorname{Re}_{x}\right)^{-1 / 2} f^{\prime \prime}(0) \\
N u=\frac{q_{w} x}{\kappa\left(T_{w}-T_{\infty}\right)}=-\left(\operatorname{Re}_{x}\right)^{1 / 2} \theta^{\prime}(0) \\
S h=\frac{M_{w} x}{D_{m}\left(C_{w}-C_{\infty}\right)}=-\left(\operatorname{Re}_{x}\right)^{1 / 2} \phi^{\prime}(0)
\end{gathered}
$$

where $\operatorname{Re}_{x}$ is the Reynolds number. And hence the values proportional to the skin-friction coefficient, Nus- selt number and Sherwood number are $f^{\prime \prime}(0),-\theta^{\prime}(0)$ and $-\phi^{\prime}(0)$ respectively.

\section{Numerical Computation}

The numerical solutions of the non-linear differential Equations (11) to (13) under the boundary conditions (14) have been performed by applying a shooting method namely Nachtsheim and Swigert [21] iteration technique (guessing the missing values) along with sixth order Runge-Kutta iteration scheme. We have chosen a step size $\Delta \eta=0.01$ to satisfy the convergence criterion of $10^{-6}$ in all cases. The value of $\eta_{\infty}$ has been found to each iteration loop by $\eta_{\infty}=\eta_{\infty}+\Delta \eta$. The maximum value of $\eta_{\infty}$ to each group of parameters $f_{w}, \gamma, \gamma^{*}, \alpha$, $M, D a$, Re, Fs, Pr, N,Ec, Sr, Du, Q, Ec, Pn and Sc has been determined when the values of the unknown boundary conditions at $\eta=0$ not change to successful loop with error less than $10^{-6}$. In order to verify the effects of the step size $\Delta \eta$, we have run the code for our model with three different step sizes as $\Delta \eta=0.01$, $\Delta \eta=0.005$ and $\Delta \eta=0.001$, and in each case we have found excellent agreement among them shown in Figures 2 to 4 .

To assess the accuracy of the present numerical method, we have compared our surface mass flux with Samad and Mohebujjaman [4] for viscous fluid when $D a$ $=1000, N=0, \alpha=0^{0}, F s=0, \quad \gamma_{x}^{*}=0, D u=0, E c=0$ and $S r=0$ and we have found excellent agreement among the results shown in Table 1. Therefore, the developed code can be used with great confidence to study the problem considered in this paper.

\section{Results and Discussion}

For the purpose of discussing the results of the flow field

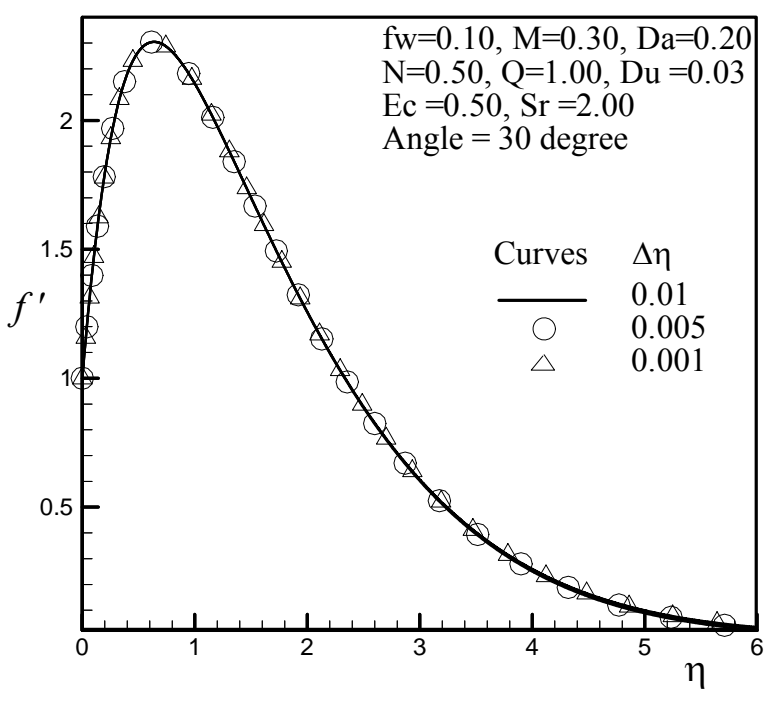

Figure 2. Velocity profiles for different step size. 


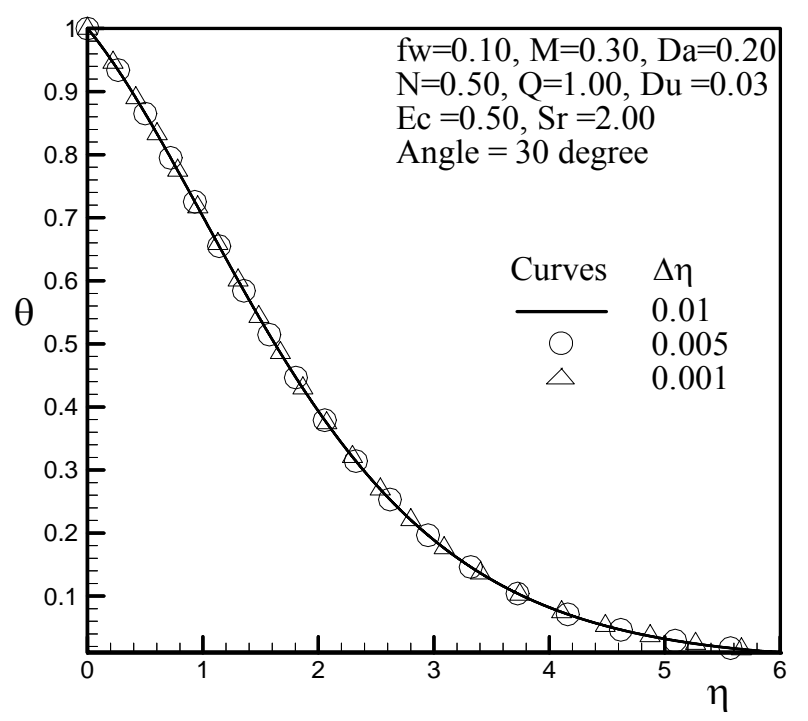

Figure 3. Temperature profiles for different step size.

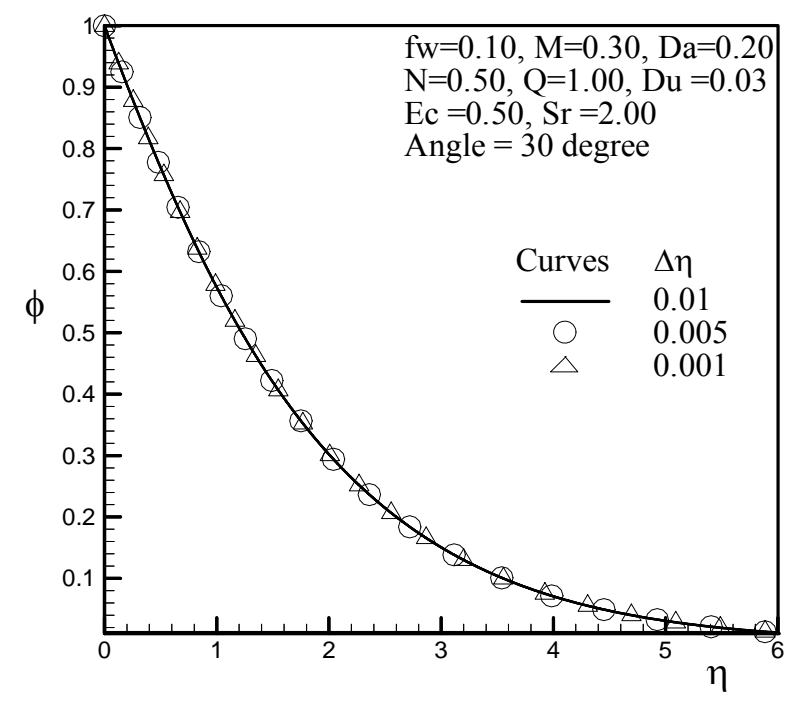

Figure 4. Concentration profiles for different step size.

Table 1. Comparison of the values of Sherwood number (Sh).

\begin{tabular}{|c|c|c|c|c|}
\hline \multicolumn{2}{|c|}{ Parameters } & \multirow{2}{*}{$\begin{array}{c}\text { Samad and } \\
\text { Mohebujjaman [4] }\end{array}$} & \multirow{2}{*}{ Present Paper } & \multirow{2}{*}{ Error (\%) } \\
\hline $\operatorname{Pr}$ & $S c$ & & & \\
\hline 0.71 & 0.22 & 0.4070962 & 0.406722 & 0.09200 \\
\hline 1.0 & 0.22 & 0.3945218 & 0.392459 & 0.52561 \\
\hline 7.0 & 0.6 & 0.8167032 & 0.819936 & 0.39427 \\
\hline 7.0 & $Q=0.75$ & 0.3495120 & 0.348537 & 0.27974 \\
\hline
\end{tabular}

represented in the Figure 1, the numerical calculations are presented in the form of non-dimensional velocity, temperature and concentration profiles. The values of buoyancy parameter $\gamma$ and modified buoyancy parameter $\gamma^{*}$ are taken to be both positive to represent cooling of the plate. The parameters are chosen arbitrarily where $\operatorname{Pr}=0.71$ corresponds physically to air at $20^{\circ} \mathrm{C}$, $\operatorname{Pr}=1.0$ corresponds to electrolyte solution such as salt water and $\operatorname{Pr}=7.0$ corresponds to water, and $S c=0.22$, 0.6 and 1.0 corresponds to hydrogen, water vapor and methanol respectively at approximate $25^{\circ} \mathrm{C}$ and 1 atmosphere. The values of Dufour number and Soret number are chosen in such a way that their product is constant provided that the mean temperature $T_{m}$ is kept constant as well.

Due to free convection problem positive large values of $\gamma_{x}=12$ and $\gamma_{x}^{*}=6$ are chosen. The value of Re is kept $100, F s$ equal to $0.09, D a=0.2, E c=0.5, f_{w}=0.1$ and $N=0.5$. However, numerical computations have been carried out for different values of the magnetic field parameter $(M)$, angle of inclination $(\alpha)$, heat source/ sink parameter $(Q)$, Dufour number $(D u)$ and Soret number $(S r)$. The numerical results for the velocity, temperature and concentration profiles are displayed in Figures 5-19.

Figures 5 to 7 display the effects of the Soret number $\mathrm{Sr}$ on the velocity, temperature and concentration profiles. It is observed that, when $\mathrm{Sr}$ increases, the velocity increases slightly while temperature decreases to a small extent. Figure 7 reveals that the Soret number $\mathrm{Sr}$ has influence on concentration profiles to a great extent. The concentration decreases near the wall first and after that starts to increase from $\eta=0.59$, due to the effect of $\mathrm{Sr}$ on the stretching sheet.

From Figure 8, we observe that first the velocity decreases rapidly and after $\eta=2.74$ starts to increase slowly with the increase of $M$. This is due to the effect of magnetic field on the stretching sheet. In addition, the temperature field as well as concentration increases with the increase of magnetic field. The magnetic field lines

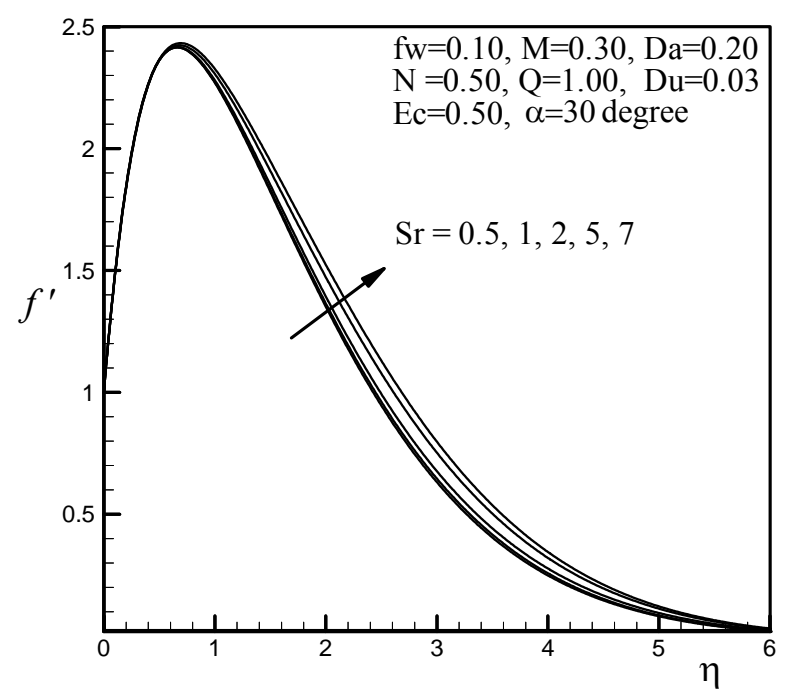

Figure 5. Velocity profiles for different values of $\boldsymbol{S r}$. 


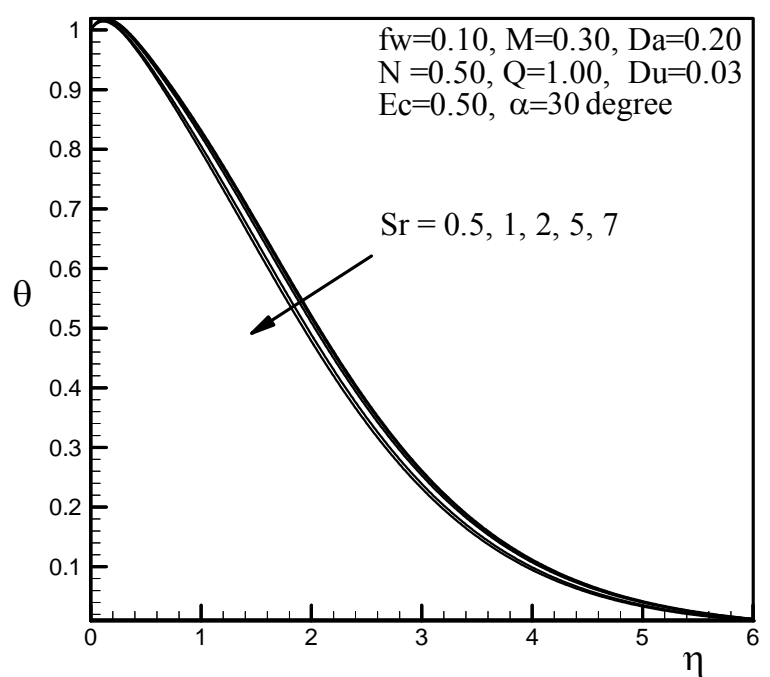

Figure 6. Temperature profiles for different values of $\boldsymbol{S r}$.

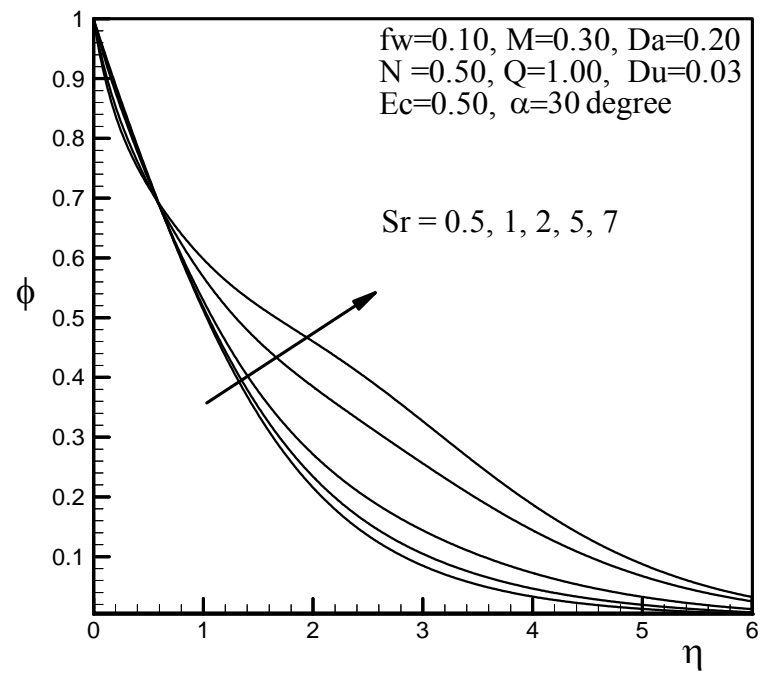

Figure 7. Concentration profiles for different values of $\boldsymbol{S r}$.

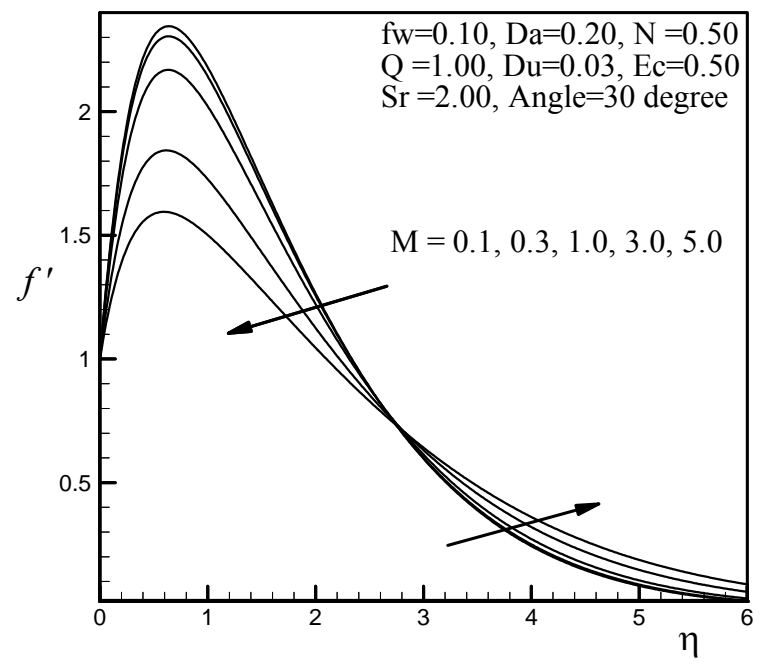

Figure 8. Velocity profiles for different values of $M$.

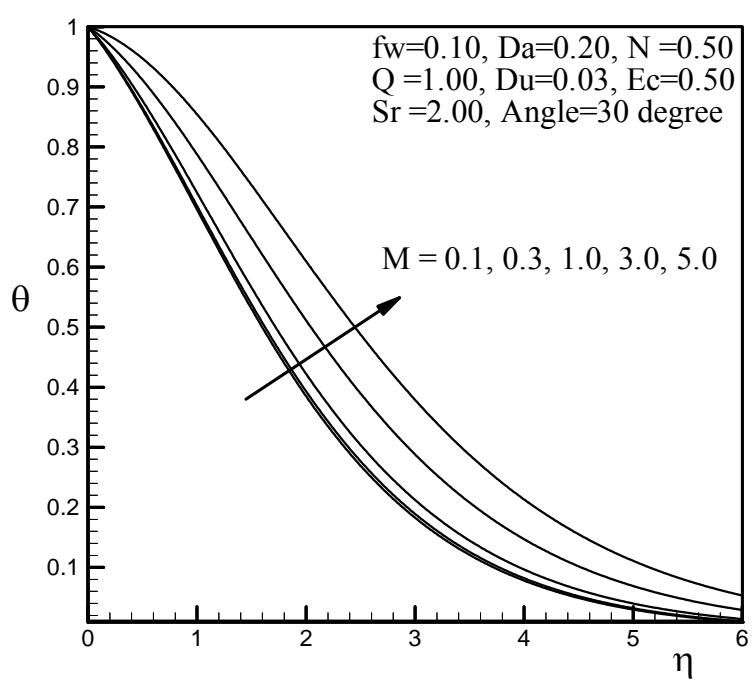

Figure 9. Temperature profiles for different values of $M$.

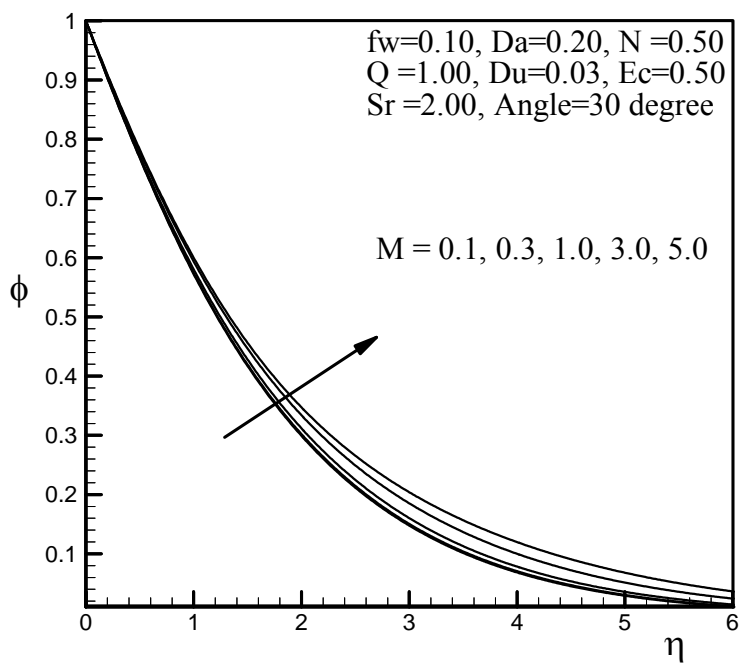

Figure 10. Concentration profiles for different values of $M$.

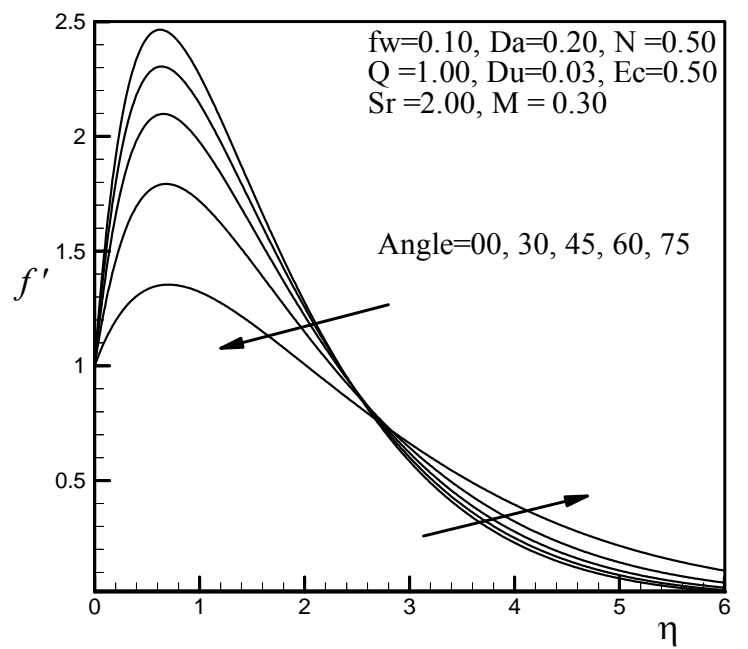

Figure 11. Velocity profiles for different values of angle $\alpha$. 


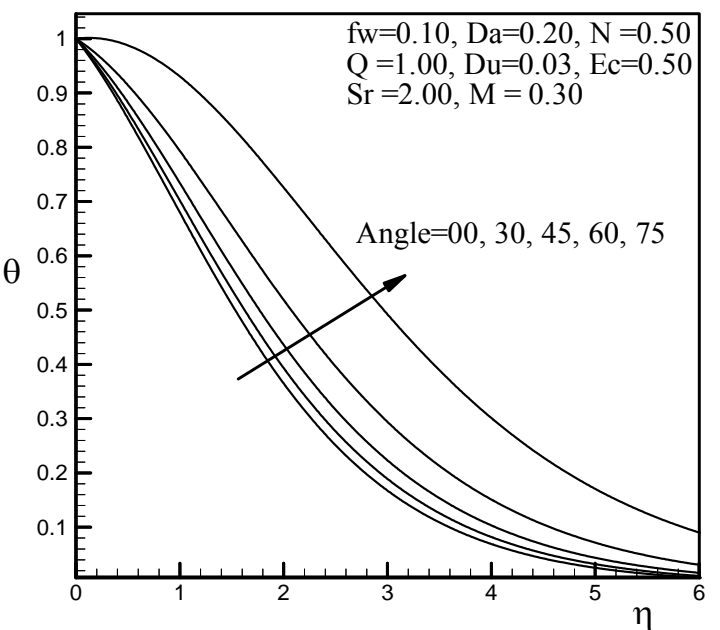

Figure 12. Temperature profiles for different values of angle $\alpha$.

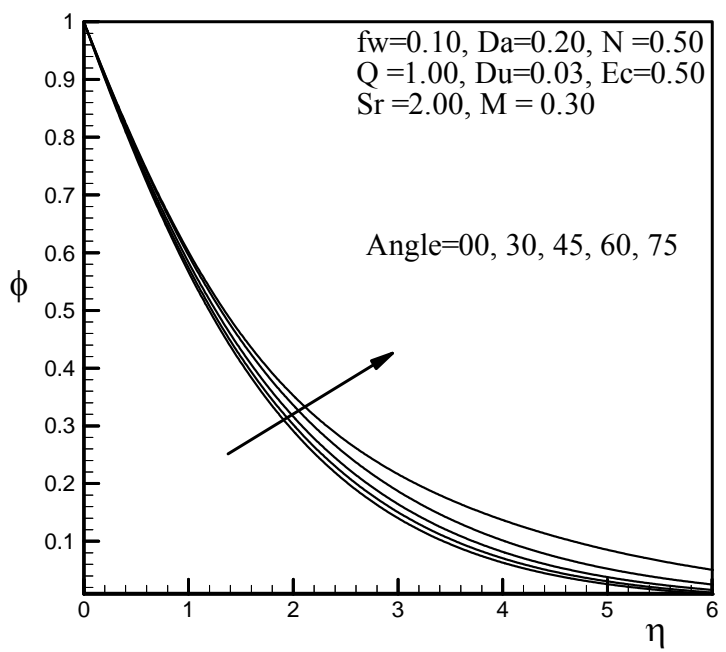

Figure 13. Concentration profiles for different values of angle $\alpha$.

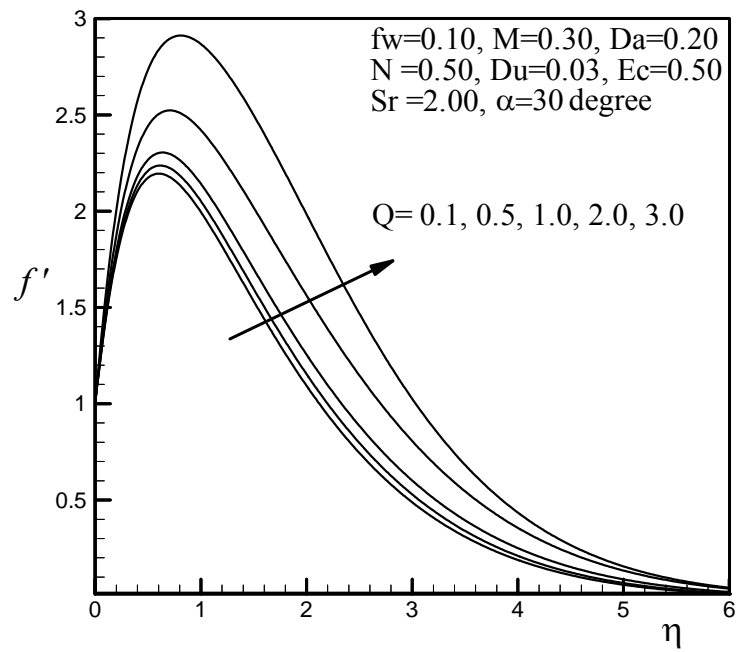

Figure 14. Velocity profiles for different values of $Q$.

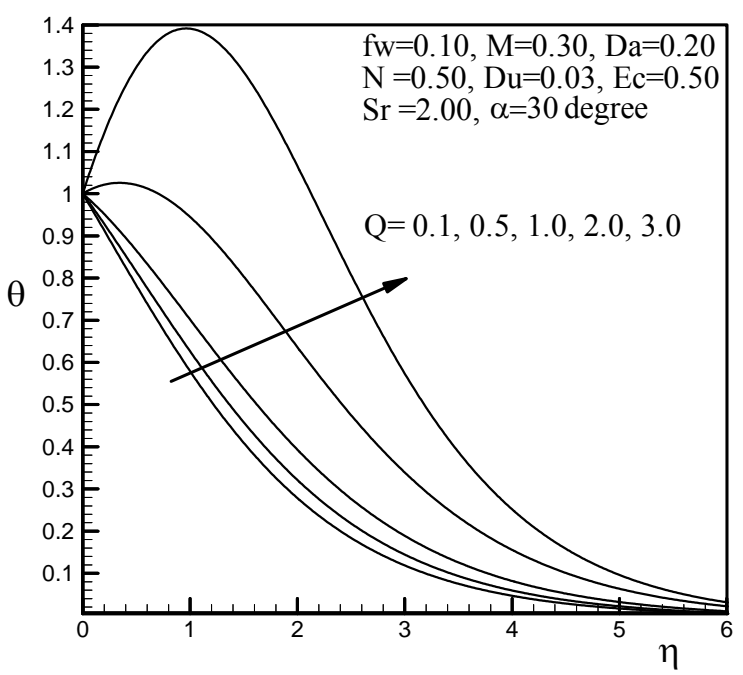

Figure 15. Temperature profiles for different values of $Q$.

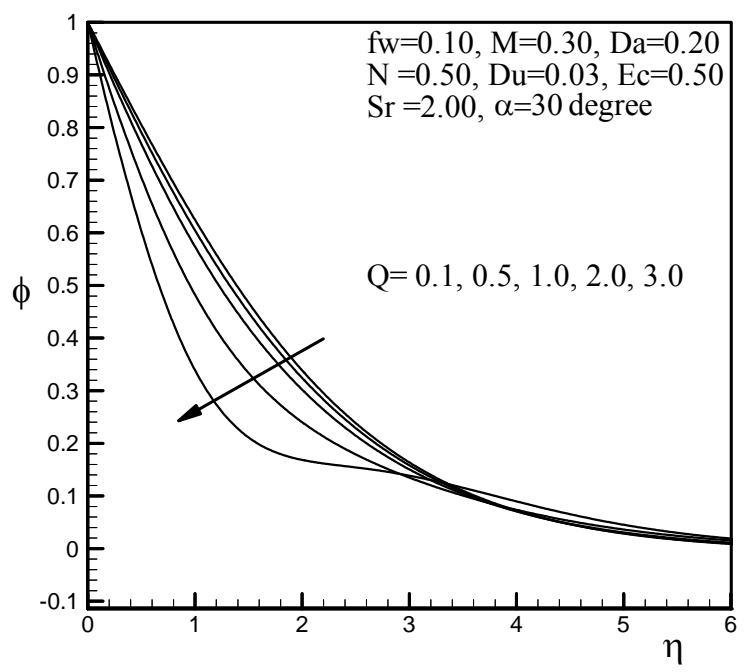

Figure 16. Concentration profiles for different values of $Q$.

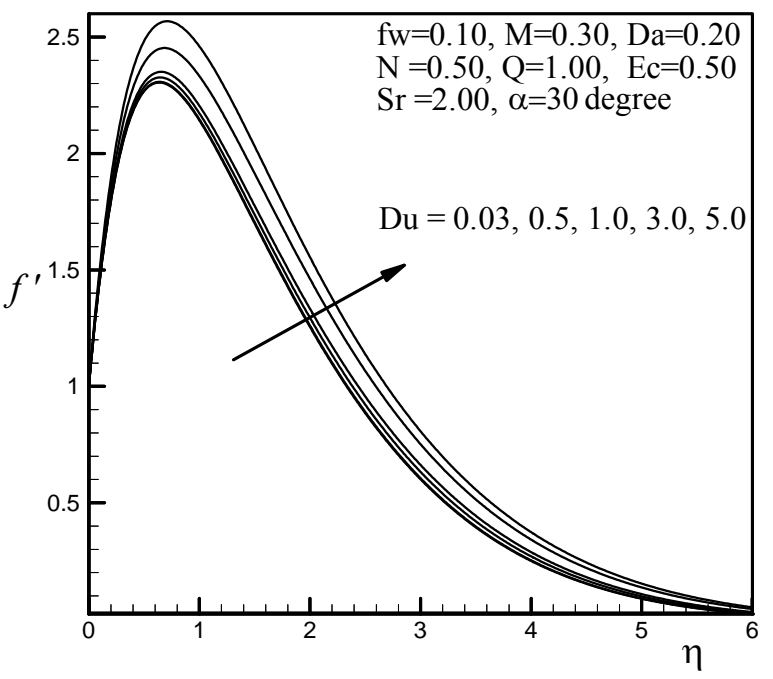

Figure 17. Velocity profiles for different values of $D u$. 


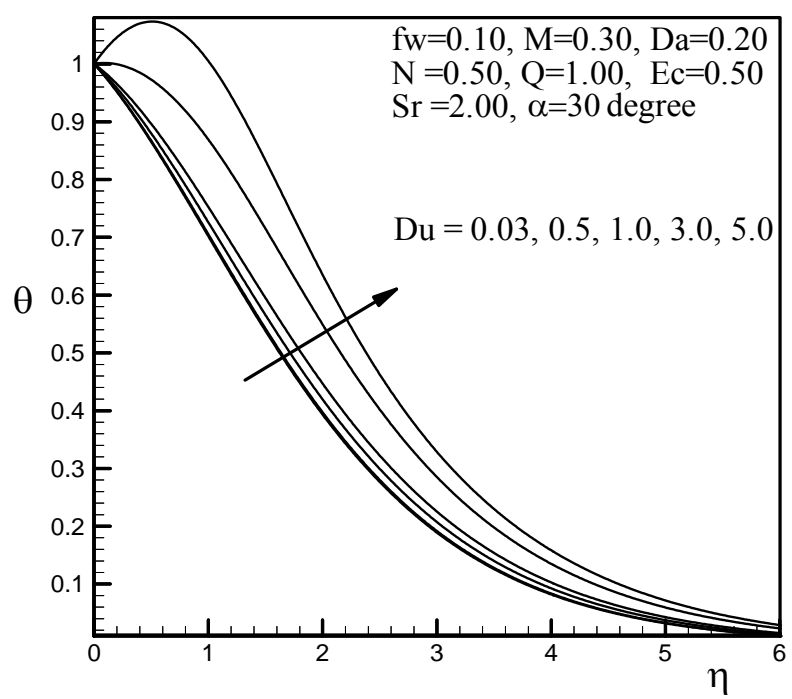

Figure 18. Temperature profiles for different values of $D u$.

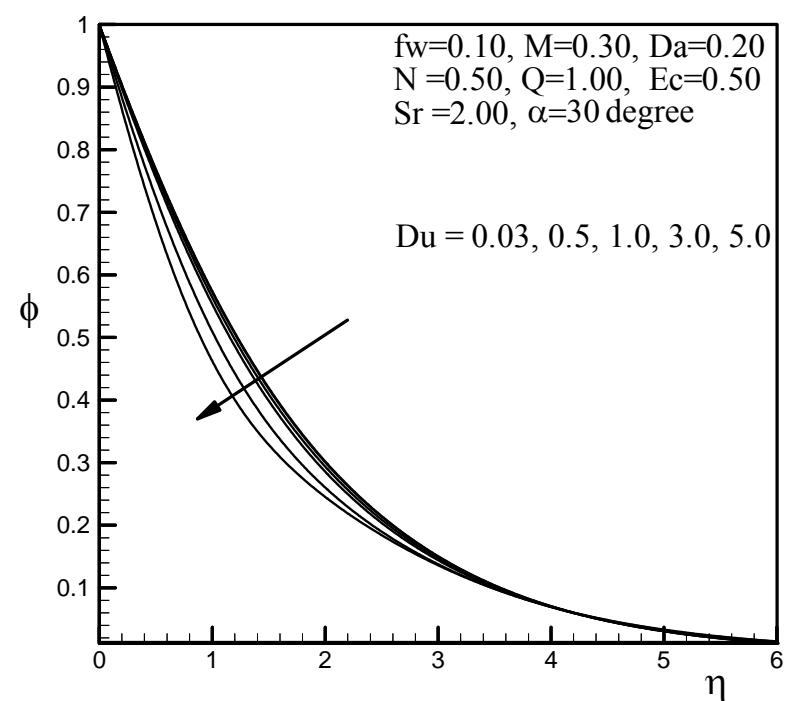

Figure 19. Concentration profiles for different values of $\mathrm{Du}$.

act like a string and tend to retard the motion of the fluid. The consequence of which is to increase the rate of heat transfer temperature variation is prominent as seen in Figure 9.

The effect of the angle of inclination $\alpha$ of the stretching sheet on the velocity field is shown in Figure 11. From this figure we see that the velocity decreases with the increase of $\alpha$ swiftly up to $\eta=2.69$. As $\alpha$ increases, the effect of the buoyancy force decreases because of the multiplication factor $\cos \alpha$ and the velocities decrease. But after $\eta=2.69$ it is seen that the velocity is increasing with the raise of $\alpha$. Figure 12 shows that the thermal boundary layer thickness and the temperature rise hastily with grow up of $\alpha$. Finally, we observe that the angle of inclination affects the concentration very slowly near the plate surface in Figure 13.
Away from the plate, however, the effect on the concentration profiles is significant.

For different values of heat source parameter $Q$ the velocity, temperature and concentration profiles are exposed in Figures 14 to 16. We have illustrated non-dimensional velocity, temperature and concentration profiles against $\eta$ for some representative values of the heat source parameter $Q=0.1,0.5,1.0,2.0,3.0$. The positive value of $Q$ represents source i.e. heat generation in the fluid. From Figure 14, it is observed that due to the generation of heat the buoyancy force increases which in turn gives higher velocity in the boundary layer. For heat generation, the peak velocity occurs near the surface of the stretching plate. This is corroborated by Figure 15 where it is seen that the temperatures do indeed rapid increase as $Q$ increases. The temperature gradient at the surface is decreasing from negative to positive as we increase $Q$. This means that when $Q=0.1$ the temperature is transferred from the sheet, but for increasing $Q$, the heat transfer rate is decreasing, when $Q=Q_{(c r t)}=1.644035$ the temperature gradient at the sheet is zero, if we further increase $Q$, the temperature gradient increases to positive value, that is, the stretching sheet gains temperature from the environment. The hydrodynamic and thermal boundary layers thickness increase but the concentration boundary layer thickness reduces with the increase of $Q$ for $\eta<2.87$. After this there is no variation but for large value of $Q$ there exist some fluctuations in the concentration profiles shown in Figure 16.

It is observed that with the increase of the value of $D u$ the velocity profiles occur higher. From Figure 18, it is seen that $D u$ has remarkable effect on temperature profiles; quantitatively when $\eta=1.5 \mathrm{Du}$ increases from 0.03 to 0.5 and there is $5.11 \%$ increase in the temperature value, whereas the corresponding increase is $16.53 \%$, when $D u$ increases from 3.0 to 5.0. The Dufour number has a falling effect on the concentration field shown in Figure 19. Quantitatively when $\eta=1.5$ and $D u$ increases from 0.03 to 0.5 , there is $2.45 \%$ decrease in the concentration value, whereas the corresponding decrease is $8.840 \%$ when $D u$ increases from 3.0 to 5.0 .

Finally, the effects of various parameters on the skin friction $C_{f}$, local Nusselt number $N u$ and local Sherwood number $S h$ are shown in the Tables 2 to $\mathbf{5 .}$

\section{Conclusions}

From the present study we can make the following conclusions:

1) Using magnetic field we can control the heat and mass transfer flow characteristics;

2) The effect of heat source parameter is very noteworthy; 
Table 2. $C_{f}$, Nu and $S h$ for different values of $\alpha$.

\begin{tabular}{cccc}
\hline$\alpha$ & $C_{f}$ & $N u$ & $S h$ \\
\hline 0 & 6.062515 & 0.236963 & 0.504611 \\
30 & 5.273891 & 0.215677 & 0.494566 \\
45 & 4.303304 & 0.183435 & 0.482631 \\
60 & 2.964883 & 0.121971 & 0.468041 \\
75 & 1.248916 & -0.024570 & 0.461948 \\
\hline
\end{tabular}

Table 3. $C_{f}, N u$ and $S h$ for different values of $Q$.

\begin{tabular}{cccc}
\hline$Q$ & $C_{f}$ & $N u$ & $S h$ \\
\hline 0.1 & 5.054863 & 0.427374 & 0.400008 \\
0.5 & 5.140483 & 0.342477 & 0.438317 \\
1.0 & 5.273890 & 0.215677 & 0.494566 \\
2.0 & 5.680986 & -0.155498 & 0.650992 \\
3.0 & 6.367314 & -0.793667 & 0.893791 \\
\hline
\end{tabular}

Table 4. $C_{f}$, Nu and $S h$ for different values of $D u$.

\begin{tabular}{cccc}
\hline$D u$ & $C_{f}$ & $N u$ & $S h$ \\
\hline 0.03 & 5.273890 & 0.215677 & 0.494566 \\
0.5 & 5.314538 & 0.181994 & 0.509765 \\
1.0 & 5.359120 & 0.143969 & 0.526497 \\
3.0 & 5.553940 & -0.036574 & 0.602589 \\
5.0 & 5.786638 & -0.286902 & 0.704735 \\
\hline
\end{tabular}

Table 5. $C_{f}, N u$ and $S h$ for different values of $S r$.

\begin{tabular}{cccc}
\hline$S r$ & $C_{f}$ & $N u$ & $S h$ \\
\hline 0.5 & 5.547109 & -0.315220 & 0.578838 \\
1.0 & 5.540321 & -0.310809 & 0.626052 \\
2.0 & 5.528690 & -0.302892 & 0.718961 \\
5.0 & 5.503159 & -0.284142 & 0.990018 \\
7.0 & 5.492415 & -0.275176 & 1.167484 \\
\hline
\end{tabular}

3) The inclination of plate has remarkable effect on temperature;

4) The Dufour effect is significant;

5) The Soret number plays a role on concentration.

\section{REFERENCES}

[1] R. S. R. Gorla, "Unsteady Mass Transfer in the Boundary Layer on a Continuous Moving Sheet Electrod," Journal of the Electrochemical Society, Vol. 125, No. 6, 1978, pp.
865-869. doi:10.1149/1.2131569

[2] D. T. Chin, "Mass Transfer to a Continuous Moving Sheet Electrode," Journal of the Electrochemical Society, Vol. 122, No. 5, 1975, pp. 643-646. doi:10.1149/1.2134281

[3] L. E. Erickson, L. T. Fan and V. G. Fox, "Heat and Mass Transfer on a Moving Continuous Flat Plate with Suction or Injection," Industrial Engineering and Chemical Fundamentals, Vol. 5, 1966, pp. 19-25. doi:10.1021/i160017a004

[4] M. A. Samad and M. Mohebujjaman, "MHD Heat and Mass Transfer Free Convection Flow along a Vertical Stretching Sheet in Presence of Magnetic Field with Heat Generation," Research Journal of Applied Science, Engineering and Technology, Vol. 1, No. 3, 2009, pp. 98-106.

[5] B. C. Sakiadis, "Boundary-Layer Behavior on Continuous Solid Surfaces: I. Boundary-Layer Equations for TwoDimensional and Axisymmetric Flow," AIChE Journal, Vol. 7, No. 1, 1961, pp. 26-28. doi:10.1002/aic.690070108

[6] R. D. Cess, "The Interaction of Thermal Radiation with Free Convection Heat Transfer," International Journal of Heat Mass Transfer, Vol. 9, No. 11, 1966, pp. 1269-1277. doi:10.1016/0017-9310(66)90119-0

[7] L. J. Crane, "Flow Past a Stretching Sheet," Zeitschrift für Angewandte Mathematik und Physik (ZAMP), Vol. 21, No. 4, 1970, pp. 645-647. doi:10.1007/BF01587695

[8] E. M. Sparrow, "Radiation Heat Transfer," Augmented Edition, Hemisphere Publishing Corp., Washington DC, 1978.

[9] V. M. Soundalgekar, N. V. Vighnesam and I. Pop, "Combined Free and Forced Convection Flow Past a Vertical Porous Plate," International Journal of Energy Research, Vol. 5, No. 3, 1981, pp. 215-226. doi:10.1002/er.4440050303

[10] W. H. H. Banks, "Similarity Solutions of the Boundary Layer Equation for a Stretching Wall," Journal de Mecanique Theorique et Appliquee, Vol. 2, No. 3, 1983, pp. 375-392.

[11] J. B. McLeod and K. R. Rajagopal, "On the Uniqueness of Flow of a Navier-Stokes Fluid Due to a Stretching Boundary," Archive for Rational Mechanics and Analysis, Vol. 98, No. 4, 1987, pp. 386-395. doi:10.1007/BF00276915

[12] C. K. Chen and M. I. Char, "Heat Transfer of a Continuous, Stretching Surface with Suction or Blowing," Journal of Mathematical Analysis and Applications, Vol. 135, No. 2, 1988, pp. 568-580. doi:10.1016/0022-247X(88)90172-2

[13] M. A. Hossain and H. S. Takhar, "Radiation Effect on Mixed Convection along a Vertical Plate with Uniform Surface Temperature," Heat and Mass Transfer, Vol. 31, No. 4, 1996, pp. 243-248. doi:10.1007/BF02328616

[14] E. R. G. Eckert and R. M. Drake, "Analysis of Heat and Mass Transfer," McGraw-Hill, New York, 1972.

[15] M. A. Alabraba, A. R. Bestman and A. Ogulu, "Laminar Convection in Binary Mixed of Hydromagnetic Flow with Radiative Heat Transfer," Astrophysics and Space 
Science, Vol. 195, No. 2, 1992, pp. 431-439. doi:10.1007/BF00646774

[16] A. Postelnicu, "Influence of a Magnetic Field on Heat and Mass Transfer by Natural Convection from Vertical Surfaces in Porous Media Considering Soret and Dufour Effects," International Journal of Heat Mass Transfer, Vol. 47, No. 6-7, 2004, pp. 1467-1472. doi:10.1016/i.ijheatmasstransfer.2003.09.017

[17] M. S. Alam and M. M. Rahman, "Dufour and Soret Effects on Mixed Convection Flow Past a Vertical Porous Flat Plate with Variable Suction," Nonlinear Analysis: Modelling and Contral, Vol. 11, No. 1, 2006, pp. 3-12.

[18] M. Enamul Karim, M. A. Samad and Md. Abdus Sattar, "Steady MHD Free Convection Flow with Thermal Radiation Past a Vertical Porous Plate Immersed in a Porous Medium," Research Journal of Mathematics and Statis- tics, Vol. 3, 2011, pp. 141-147.

[19] K. A. Helmy, "MHD Boundary Layer Equations for Power Law Fluids with Variable Electric Conductivity," Meccanica, Vol. 30, No. 2, 1995, pp. 187-200. doi:10.1007/BF00990456

[20] K. Vajravelu and A. Hadjinicolaou, "Heat Transfer in a Viscous Fluid over a Stretching Sheet with Viscous Dissipation and Internal Heat Generation," International Communications in Heat Mass Transfer, Vol. 20, No. 3, 1993, pp. 417-430. doi:10.1016/0735-1933(93)90026-R

[21] P. R. Nachtsheim and P. Swigert, "Satisfaction of the Asymptotic Boundary Conditions in Numerical Solution of the Systems of Non-Linear Equations of Boundary Layer Type," Ph.D. Thesis, NASA TN D-3004, Washington DC, 1965. 